\title{
Toxoplasmosis congénita: Diagnóstico serológico, RPC, aislamiento y caracterización molecular de Toxoplasma gondii
}

\author{
Liliana Carral, Federico Kaufer, Lais Pardini, Ricardo Durlach, \\ Gastón Moré, María C. Venturini y Cristina Freuler
}

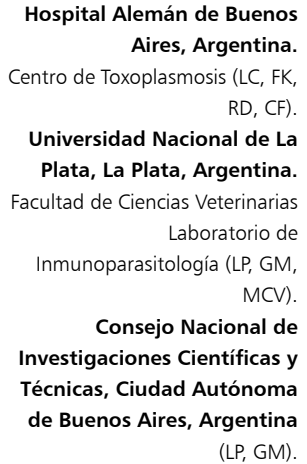

Los autores declaran que no existieron conflictos de interés en el presente estudio. No hubo fuentes de financiación externas.

Recibido: 20 de junio de 2017 Aceptado: 4 de enero de 2018

Correspondencia a: Liliana Ángela Carral lacarral@yahoo.es

\section{Introducción}

L a infección por Toxoplasma gondii en el embarazo puede producir graves consecuencias en el feto, como muerte intrauterina, corio-retinitis, hidrocefalia, calcificaciones cerebrales, microcefalia, microftalmia, ceguera, retraso mental o psicomotor. La gravedad de las secuelas de la toxoplasmosis congénita (TC) está asociada a la edad del feto en el momento de la infección materna. Los niños nacidos de una madre infectada en el primer trimestre tienen alta probabilidad de estar gravemente afectados, mientras que $80 \%$ de los niños que se infectan durante el tercer trimestre nacen asintomáticos. Todos los recién nacidos (RN) con TC requieren tratamiento, incluyendo los que nacen asintomáticos para evitar el riesgo de desarrollo de secuelas tardías ${ }^{1}$.

El diagnóstico serológico del RN se hace con la investigación de las inmunoglobulinas $\operatorname{IgG}, \operatorname{IgM}$, IgA e IgE específicas en sangre. Las IgM, IgA e IgE no atraviesan la placenta, su detección indica producción fetal y se las utiliza como marcadores serológicos de infección congénita. La IgG atraviesa la placenta y en los niños no infectados, los títulos de las IgG (de origen materno), descienden hasta su desaparición total. Por el contrario, en los niños infectados los títulos se mantienen o aumentan y ofrecen un elemento diagnóstico fundamental. Al año del nacimiento el diagnóstico es definitivo, el niño será considerado libre de infección cuando las IgG específicas no sean detectables en su sangre. La persistencia de las IgG confirma la infección prenatal ${ }^{2}$.

La técnica más sensible para la determinación de $\operatorname{IgM}, \operatorname{IgA}$ e IgE es immunosorbent agglutination assay (ISAGA) ${ }^{3}$. El estudio serológico simultáneo de la sangre materna y del RN ofrece la utilidad diagnóstica comparativa; si alguno de los anticuerpos IgM, IgA o IgE está presente en la sangre del niño y no en la de la madre, confirma la infección congénita.

El estudio serológico debe ser completado con la metodología directa que incluye el aislamiento del parásito y la reacción de polimerasa en cadena (RPC) en la placenta, sangre de cordón y/o sangre del $\mathrm{RN}^{4}$.

El objetivo del presente estudio fue evaluar los métodos utilizados en nuestro laboratorio para el diagnóstico de TC en los neonatos nacidos de madres que cursaron una infección aguda durante el embarazo.

Debido a que la patogenicidad de la infección podría estar asociada a diferentes genotipos de Toxoplasma gondii ${ }^{5}$ se realizó un estudio experimental en conjunto 
con el Laboratorio de Inmunoparasitología (LAINPA) de la Facultad de Ciencias Veterinarias de la Universidad Nacional de La Plata para la caracterización molecular de los aislamientos obtenidos.

\section{Materiales y Métodos}

Se incluyeron 67 niños RN de madres a quienes se diagnosticó toxoplasmosis aguda durante el embarazo en el Centro de Toxoplasmosis del Hospital Alemán de Buenos Aires (CTHA) entre enero de 2006 y marzo de 2016. Cuatro casos fueron pesquisados mediante el programa de prevención de la TC implementado en el Hospital Alemán donde se realizan controles serológicos trimestrales a las embarazadas. El resto de los casos corresponden a derivaciones de otros centros cuando las pruebas de tamizaje sugirieron una infección aguda y se derivaron al CTHA para la confirmación diagnóstica y seguimiento.

El estudio utilizó el algoritmo diagnóstico para el RN del Consenso Argentino para la Prevención de la Toxoplasmosis Congénita de 2005 que incluye la determinación de $\operatorname{IgG}$, la detección de $\operatorname{IgM}$, IgA e $\operatorname{IgE}$ y la comparación con la serología materna. Además, se incluyó el aislamiento del parásito a partir de placenta o sangre de cordón y la RPC ${ }^{4}$.

El estudio serológico utilizó para la determinación de $\mathrm{IgG}$ la reacción de Sabin Feldman $(\mathrm{SF})^{6}$, método de referencia para toxoplasmosis y la técnica de la ISAGA para la detección de IgM, IgA e IgE. Cuando alguna de las reacciones con la técnica de ISAGA fue positiva se repitió el estudio, con un lapso mínimo de 10 días, para descartar la contaminación con sangre materna ${ }^{7-9}$.

En 18 casos se realizó RPC en tiempo real (RPC-TR) en sangre de cordón umbilical mediante la amplificación de una secuencia repetitiva de la región del gen B1 del genoma de $T$. gondii ${ }^{10}$.

Veintiuna placentas y 20 muestras de sangre de cordón umbilical (SC) se procesaron según el procedimiento descrito por $\mathrm{MH}$ Bessieres ${ }^{11}$ para el aislamiento del parásito. Se consideraron positivos si se detectaron anticuerpos específicos en suero de ratón mediante la reacción de SF y/o se observaron quistes del protozoo en el cerebro. Los materiales de los aislamientos positivos se enviaron al LAINPA para su caracterización molecular. Se conservaron por pasaje en ratones GKO para interferón gamma, cultivo celular y criopreservación en nitrógeno líquido.

Adicionalmente se realizó una prueba de RPC utilizando los partidores TOX5-TOX8 que permitió detectar ADN específico del parásito. Los aislamientos se caracterizaron por nPCR-RFLP (RPC anidada seguida por cortes con enzimas de restricción) para nueve marcadores (nSAG2, SAG3, BTUB, GRA6, c29-2, c22-8, L358, PK1 y APICO), procediendo como se describiera en estudios previos $^{12}$

El diagnóstico de TC se consideró positivo si se cumplieron una o varias de las siguientes condiciones:

- uno de los marcadores serológicos (IgM, IgA o IgE) presente en dos muestras consecutivas en suero con diez días de diferencia, o

- un aislamiento positivo y/o la IgG permanecía positiva al año.

La infección prenatal se descartó en los niños que presentaron marcadores serológicos y aislamiento negativos al nacimiento y que por protocolo fueron seguidos con la serología hasta la desaparición total de las IgG.

Los RN fueron evaluados clínicamente, con fondo de ojo y ecografía cerebral.

\section{Resultados}

La reacción de SF en los $67 \mathrm{RN}$ incluidos en el estudio tuvo títulos iguales o mayores a 1/4.096.

En 43 niños la reacción de SF se tornó negativa durante el seguimiento serológico en el primer año de vida y se los consideró libres de infección (grupo LI). Ninguno de ellos presentó IgM, IgA o IgE positiva ni al nacimiento ni en controles posteriores. El estudio del grupo LI incluyó el procesamiento de 20 placentas y 16 muestras de sangre de cordón umbilical para aislamiento del parásito, los cuales fueron todos negativos, y la realización de RPC-TR que se llevó a cabo en 16 muestras de sangre de cordón, en 14 casos fue negativa y positiva en dos.

El diagnóstico de TC se hizo en $24 \mathrm{RN}$, (grupo TC). En $20 \mathrm{RN}$ del grupo TC la ISAGA IgM e IgA fueron positivas, en un RN sólo fue positiva la $\operatorname{IgM}$ y en otros dos sólo la IgA. La búsqueda de $\operatorname{IgE}$ se realizó a 14 de los $24 \mathrm{RN}$ y seis resultaron positivos, en un caso la IgE fue positiva en el niño y negativa en la madre. Un caso de TC presentó los tres marcadores serológicos negativos y fue diagnosticado sólo con el aislamiento.

La técnica de RPC-TR se realizó sólo en dos RN del grupo TC y uno fue positivo.

Con el propósito de aislar el parásito se procesaron una placenta y cuatro muestras de sangre de cordón. En la placenta y en tres muestras de sangre de cordón se aisló T. gondii. En la muestra de sangre de cordón restante el aislamiento fue negativo, la muestra no fue obtenida al nacimiento sino siete días después, cuando el niño ya estaba en tratamiento.

La genotipificación del protozoario a partir de la placenta resultó tipo clonal $\mathrm{II}^{12}$ y los tres aislamientos de sangre de cordón umbilical fueron "atípicos", uno tipo III para todos los marcadores excepto para C29-2 (tipo I), los otros dos aislamientos fueron tipo III para la mayoría 


\begin{tabular}{|c|c|c|c|c|}
\hline & $S(n)$ & $E(n)$ & $\operatorname{VPP}(n)$ & VPN (n) \\
\hline ISAGA M & $87 \%(21 / 24)$ & $100 \%(43 / 43)$ & $100 \%(21 / 21)$ & $93 \%(43 / 46)$ \\
\hline ISAGA A & $91 \%(22 / 24)$ & $100 \%(43 / 43)$ & $100 \%(22 / 22)$ & $95 \%(43 / 46)$ \\
\hline ISAGA E & $43 \% \quad(6 / 14)$ & $100 \%(22 / 22)$ & $100 \% \quad(6 / 6)$ & $73 \%(22 / 30)$ \\
\hline ISAGA M+A & $95 \%(23 / 24)$ & $100 \%(43 / 43)$ & $100 \%(23 / 23)$ & $98 \%(43 / 44)$ \\
\hline
\end{tabular}

Tabla 2. Sensibilidad (S), Especificidad (E), VPP y VPN del aislamiento del parásito

$\begin{array}{ccccc} & \mathbf{S ~ ( n )} & \mathbf{E ~ ( n )} & \mathbf{V P P}(\mathbf{n}) & \text { VPN (n) } \\ \text { Aislamiento } & 80 \%(4 / 5) & 100 \%(36 / 36) & 100 \%(4 / 4) & 97 \%(36 / 37)\end{array}$

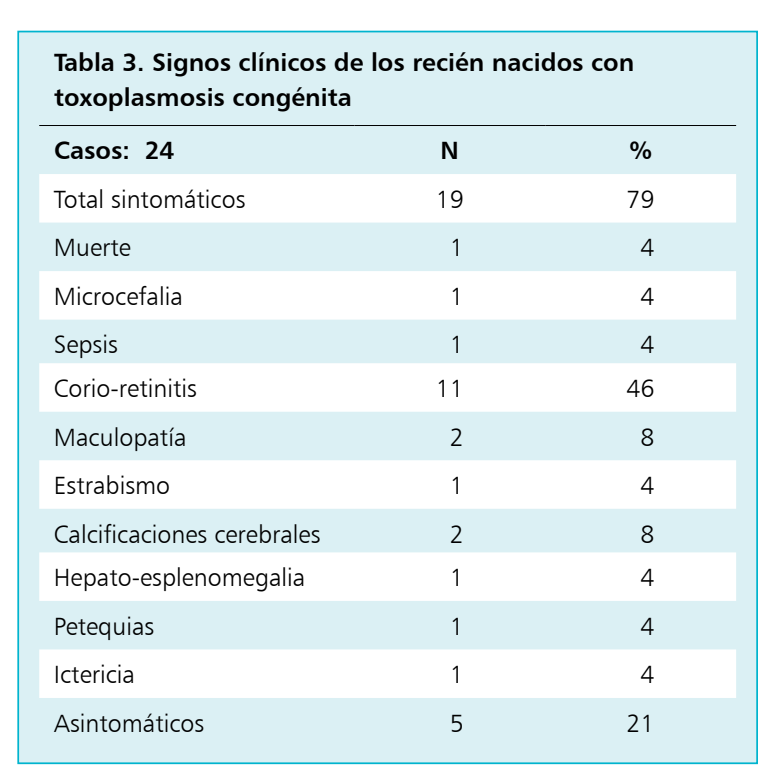

de los marcadores y uno de ellos tipo I para PK1, L358 y C22-8 y el otro aislamiento tipo II para PK1 y tipo I para L $358^{13}$.

En la Tabla 1 se muestra la sensibilidad (S), especificidad (E), valor predictor positivo (VPP) y valor predictor negativo (VPN) calculados sobre los resultados obtenidos con las reacciones serológicas y en la Tabla 2 la de los aislamientos.

En la Tabla 3 se detalla la manifestación clínica de los recién nacidos.

\section{Discusión}

La TC, consecuencia de una primoinfección intragestacional aguda, es prevenible con los programas de control serológico durante el embarazo y sus secuelas se pueden evitar o aminorar con el tratamiento adecuado. Los $\mathrm{RN}$ deben ser estudiados desde el punto de vista clínico y de laboratorio. Si se confirma la infección, deben ser tratados. Los RN sin evidencia clínica ni serológica deben ser controlados hasta la desaparición total de las IgG para poder descartar la infección congénita ${ }^{14}$.

La patogenicidad de la TC depende del período de embarazo de la infección materna, de la gravedad de la infección intrauterina, del tratamiento materno y de la virulencia del parásito actuante. En este estudio, ocho madres cursaron su primoinfección durante el $3^{\circ}$ trimestre y sólo recibieron unas pocas semanas de tratamiento; 16 de ellas no habían sido controladas y el diagnóstico se realizó en el momento del parto. En un estudio previo de nuestro grupo sobre la prevención de la TC en nueve hospitales de Buenos Aires, los casos de TC se detectaron en RN de madres que no habían tenido controles durante la gestación o en quienes el diagnóstico se efectuó en el parto o pocas semanas antes por lo cual no habían recibido tratamiento efectivo ${ }^{15}$. Estos resultados avalan la importancia de los programas de prevención de la TC.

El diagnóstico serológico del RN se realizó con la detección de $\operatorname{IgM}$ y/o IgA en sangre en $96 \%$ de los casos. La determinación de ambos marcadores fue importante ya que en dos niños sólo se pudo detectar IgA y en otro sólo IgM. Estos resultados coinciden con los obtenidos por otros autores ${ }^{16,17}$. La comparación entre la serología del neonato y la de su madre confirmó dos casos, en uno se detectó IgE positiva sólo en el suero del niño y en el otro los títulos de $\operatorname{IgM}$, IgA e IgE fueron significativamente mayores en el suero del RN en dos muestras obtenidas con 10 días de diferencia ${ }^{2}$.

Si bien la sensibilidad de la serología es alta, no alcanzó a $100 \%$; un caso fue diagnosticado sólo mediante el aislamiento del parásito en un $\mathrm{RN}$ con todos los marcadores serológicos negativos. El aislamiento es una importante herramienta para el diagnóstico de la TC; aproximadamente $10 \%$ de los pacientes con marcadores serológicos negativos son diagnosticados mediante este método ${ }^{18}$.

La RPC-TR en sangre de cordón fue aplicada en sólo dos muestras del grupo de niños con TC en nuestro estudio, y una resultó negativa. El procesamiento de mayor número de muestras permitirá estimar la sensibilidad más adecuadamente. El resultado negativo en una muestra con TC podría explicarse por una carga parasitaria baja, el tratamiento materno o una inadecuada conservación y transporte de la muestra. Los dos casos de RPC-TR positiva en el grupo de los $\mathrm{RN}$ no infectados fueron considerados como falsos positivos cuando se descartó la infección congénita al año con las IgG negativas.

Los aislamientos del parásito tuvieron una buena performance con una sensibilidad de $80 \%$ (4/5) y una especificidad de $100 \%$. En un caso fue el único marca- 
dor positivo de la infección congénita. En el paciente con infección aguda y aislamiento negativo, la toma de muestra se realizó en sangre del niño a los siete días de vida, cuando ya estaba bajo tratamiento y sugiere la importancia de tomar las muestras para serología, RPC o aislamiento lo más cercanas posible al parto y antes de iniciar el tratamiento.

En el presente estudio 19 de 24 (79\%) de los niños con TC presentaron uno o más signos clínicos y los más frecuentes fueron las lesiones oculares, la corio-retinitis $11 / 19$ (46\%), maculopatías 2/19 (8\%) y el estrabismo $1 / 19$ (4\%). Otros estudios también muestran una elevada frecuencia de signos y secuelas en niños cuyas madres no habían sido diagnosticadas durante su embarazo comparados con otra cohorte de $\mathrm{RN}$ cuyas madres tuvieron acceso a programas de prevención ${ }^{19}$.

La patogenicidad de la infección podría estar asociada al genotipo del T. gondii ${ }^{5}$ involucrado en la infección. En Europa se aisló con más frecuencia el genotipo clonal II en los casos de enfermedad congénita y la mayoría de los RN fueron asintomáticos, los aislamientos con genotipos I y III sólo fueron obtenidos en 10 y $9 \%$ de los pacientes, respectivamente. En Sudamérica (Brasil, Colombia, Guyana y Argentina) es frecuente el aislamiento de los genotipos III y atípicos, y hay evidencia que la frecuencia y gravedad es mayor, con compromiso ocular en particular ${ }^{20,21}$. El riesgo de lesiones oculares en RNs infectados en Europa es de 10\%, mientras que en Brasil es de $50 \%$ con una frecuencia de reactivaciones cuatro veces mayor ${ }^{22}$. Se postula la co-evolución hospederoparásito, dada por una larga adaptación del hospedero a un determinado tipo de parásito, para explicar la baja patogenicidad observada en Europa. Por otra parte, la alta diversidad genotípica de $T$. gondii característica de Sudamérica podría relacionarse con una menor adaptación de los hospederos, especialmente aquellos con ancestros europeos, y la observación de casos más graves ${ }^{23}$. En un estudio en conjunto del Laboratorio de Toxoplasmosis del Hospital Alemán con el LAINPA se reportó el primer aislamiento con genotipificación en Argentina a partir de placenta y fue tipo clonal $\mathrm{II}^{12}$. Tres de los aislamientos a partir de sangre de cordón fueron genotipos considerados "atípicos" y no difieren demasiado de otros aislamientos de Sudamérica. Un genotipo fue similar al identificado en ToxoDB como \#14 ó 138 y los otros dos serían genotipos nuevos de acuerdo a la ToxoDB siendo tipo III para la mayoría de los marcadores, uno de ellos tipo I para PK1, L358 y C22-8 y el otro tipo II para PK1 y tipo I para L358 ${ }^{13}$. Los aislados caracterizados en este estudio, si bien son pocos casos, sugieren que los genotipos circulantes del parásito en nuestro país serían similares a los encon- trados en el resto de Sudamérica y el alto porcentaje de secuelas observado argumenta a favor de la necesidad de controlar a nuestras mujeres embarazadas para prevenir casos de TC.

\section{Conclusión}

Los métodos serológicos utilizados mostraron una buena performance para el diagnóstico de la TC. El diagnóstico serológico tiene un alto nivel de de $\mathrm{S}$ y $\mathrm{E}$ (98 y 100\%, respectivamente). El aporte de técnicas del examen directo, RPC y el aislamiento del parásito, asociados al seguimiento serológico hasta el año de vida, es fundamental para el diagnóstico de la TC. La genotipificación está reservada a los laboratorios de investigación, tiene gran valor epidemiológico, justificando mayores estudios colaborativos e interdisciplinarios para ampliar el conocimiento del impacto de los diferentes genotipos de $T$. gondii en la salud de la población.

\section{Resumen}

Introducción: El diagnóstico de toxoplasmosis congénita (TC) en el recién nacido es muy importante porque debe recibir tratamiento siempre, sintomático o no, para evitar o aminorar las secuelas de la enfermedad. Objetivo: Evaluación comparativa de los métodos disponibles en la institución para el diagnóstico de TC. Materiales y Métodos: Se evaluaron métodos diagnósticos en 67 niños cuyas madres cursaron toxoplasmosis aguda durante el embarazo. Se utilizó la técnica de Sabin Feldman para IgG al nacimiento y durante el seguimiento serológico hasta el año de vida. Para determinar IgM, IgA e IgE se utilizó la técnica immunosorbent agglutination assay (ISAGA). El diagnóstico directo se realizó por reacción de polimerasa en cadena (RPC), aislamiento y caracterización molecular del parásito. Resultados: La sensibilidad (S) de ISAGA IgM fue $87 \%$, ISAGA IgA $91 \%$ y la especificidad (E) fue $100 \%$ para ambas; cuando se realizaron en conjunto, la $\mathrm{S}$ aumentó a 98\%. La detección de IgE contribuyó al diagnóstico cuando se la detectó sólo en la sangre del neonato y no en sangre materna. Se aisló el parásito en cuatro casos de TC, uno fue genotipo II y los otros tres, genotipos "atípicos". La S del aislamiento fue $80 \%$ y la E $100 \%$. Conclusión: Los métodos serológicos utilizados mostraron una buena eficacia diagnóstica. Un caso fue detectado sólo por el aislamiento y la caracterización molecular tiene gran valor epidemiológico. 


\section{Referencias bibliográficas}

1.- Remington J S, McLeod R, Desmonts G. Toxoplasmosis. In: Remington J S, Klein J $\mathrm{O}$ (Eds). Infectious Diseases of the Fetus and Newborn Infant. 4th ed. Philadelphia Wb Saunders 1995; 140-267. 4.

2.- Pomares C, Montoya J G. Laboratory diagnosis of congenital toxoplasmosis. J Clin Microbiol 2016; 54 (10): 244854.

3.- Pinon J M, Dumon H, Chemla C, Franck J, Petersen E, Lebech, et al. Strategy for diagnosis of congenital toxoplasmosis: evaluation of methods comparing mothers and newborns and standard methods for postnatal detection of immunoglobulin G, M, and A antibodies. J Clin Microbiol 2001; 39: 2267-71.

4.- Durlach R, Kaufer F, Carral L, Freuler C, Ceriotto M, Rodríguez M, et al. Consenso argentino de toxoplasmosis congénita. Medicina (Buenos Aires) 2008; 68: 75-87.

5.- Rico-Torres C P, Vargas-Villavicencio J A, Correa D. Is Toxoplasma gondii type related to clinical outcome in human congenital infection? Systematic and critical review. Eur J Clin Microbiol Infect Dis 2016; 35 (7): 1079-88.

6.- Sabin A B, Feldman H A. Dyes as microchemical indicators of a new immunity phenomenon affecting a protozoon parasite (Toxoplasma). Science. 1948; 108 (2815): 660-3.

7.- Desmonts G, Naot Y, Remington J S Immunoglobulin M-immunosorbent agglutination assay for diagnosis of infectious diseases: diagnosis of acute congenital and acquired Toxoplasma infections. J Clin Microbiol 1981; 14 (5): 486-91.

8.- Saathoff M, Seitz H M. Detection of toxoplasma specific IgA and IgM antibodies in serum samples of adults with acquired toxoplasma infection. Z Geburtsh Perinat 1992; 196: 221-3.

9.- Wong S Y, Hajdu M P, Ramírez R, Thulliez P, McLeod R, Remington J S. Role of specific immunoglobulin $\mathrm{E}$ in diagnosis of acute toxoplasma infection and congenital toxoplasmosis. J Clin Microbiol 1993; 31: 2952-9.

10.- Burg J L, Grover C M, Pouletty P, Boothroyd I C. Direct and sensitive detection of a pathogenic protozoan, Toxoplasma gondii, by polymerase chain reaction. J Clin Microbiol 1989; 27 (8): 1787-92.

11.- Bessières $\mathrm{M} \mathrm{H}$, Berrebi $\mathrm{A}$, Rolland $\mathrm{M}$, Bloom M C, Roques C, Cassaring S, et al. Neonatal screening for congenital toxoplasmosis in a cohort of 165 women infected during pregnancy and influence of in utero treatment on the results of neonatal tests. Eur J Obstet Gynecol Reprod Biol 2001; 94 (1): 37-45.

12.- Pardini L, Carral L A, Bernstein M, Gos M L, Olejnik P, Unzaga J M, et al. First isolation and molecular characterization of Toxoplasma gondii from a human placenta in Argentina. Parasitol Int 2014; 63 (2): 470-2.

13.- Diagnóstico de Toxoplasmosis. Experiencia en el Hospital Alemán de Buenos Aires. Liliana Carral, F. Kaufer, M Messina, V. Schneider. Centro de Toxoplasmosis del Hospital Alemán de Buenos Aires. http://www.protozoologia.org. ar/Admin/mim/Libro de resumenes SAP2016. pdf. [julio de 2017].

14.- Villena I, Ancelle T, Delmas C, García P, Brezin A P, Thulliez P, et al. Congenital toxoplasmosis in France in 2007: first results from a national surveillance system. Toxosurv network and National Reference Centre for Toxoplasmosis. Euro Surveill 2010; 24: 15

15.- Carral L, Kaufer F, Durlach R, Freuler C, Olejnik P, Nadal M, et al. Multicenter study on the prevention of congenital toxoplasmosis in
Buenos Aires. Medicina (B. Aires) 2008; 68: 417-22.

16.- Moncada P A, Montoya J G. Toxoplasmosis in the fetus and newborn: an update on prevalence, diagnosis and treatment. Expert Rev Anti Infect Ther 2012; 10 (7): 815-28.

17.- Murat J B, Souvignet A, Fricker-Hidalgo $\mathrm{H}$, Brenier-Pinchart M P, Bost-Bru C, Pelloux H. Assessment of the IgA immunosorbent agglutination assay for the diagnosis of congenital toxoplasmosis on a series of 145 toxoplasmic seroconversions. Clin Vaccine Immunol 2015; 22 (4): 456-8.

18.- Robert-Gangneux F, Dupretz P, Yvenou C, Quinio D, Poulain P, Guiguen C, et al. Clinical relevance of placenta examination for the diagnosis of congenital toxoplasmosis. Pediatr Infect Dis J 2010; 29: 33-8.

19.- Olariu T R, Remington J S, McLeod R, Alam A, Montoya J G. Severe congenital toxoplasmosis in the United States: clinical and serologic findings in untreated infants. Pediatr Infect Dis J 2011; 30 (12): 1056-61.

20.- Khan A, Jordan C, Muccioli C, Vallochi A L, Rizzo L V, Belfort R Jr, et al. Genetic divergence of Toxoplasma gondii strains associated with ocular toxoplasmosis, Brazil. Emerg Infect Dis 2006; 12: 942-9.

21.- Sauer A, de la Torre A, Gómez-Marin J, Bourcier T, Garweg J, Speeg-Schatz C, et al. Prevention of retinochoroiditis in congenital toxoplasmosis: Europe versus South America. Pediatr Infect Dis J 2011; 30 (7): 601-3.

22.- Gilbert R E, Freeman K, Lago E G, BahiaOliveira L M G, Tan H K, Wallon M, et al. Ocular sequelae of congenital toxoplasmosis in Brazil compared with Europe. PLoS Negl Trop Dis 2008: 2 (8).

23.- Robert-Gangneux F, Dardé M L. Epidemiology of and diagnostic strategies for toxoplasmosis. Clin Microbiol Rev 2012 (2): 264-96. 